\title{
Instability of thermocapillary liquid layers for Oldroyd-B fluid
}

Kai-Xin Hu, Meng He, and Qi-Sheng Chen

Citation: Physics of Fluids 28, 033105 (2016); doi: 10.1063/1.4943971

View online: http://dx.doi.org/10.1063/1.4943971

View Table of Contents: http://aip.scitation.org/toc/phf/28/3

Published by the American Institute of Physics 


\title{
Instability of thermocapillary liquid layers for Oldroyd-B fluid
}

\author{
Kai-Xin Hu, Meng He, and Qi-Sheng Chen \\ National Microgravity laboratory, Institute of Mechanics, Chinese Academy of Sciences, \\ 15 Bei Si Huan Xi Road, Beijing 100190, China
}

(Received 16 November 2015; accepted 2 March 2016; published online 18 March 2016)

\begin{abstract}
The linear stability analysis of Oldroyd-B fluid for thermocapillary liquid layers is carried out. Results are presented for linear flow and return flow with Prandtl numbers of 0.02 and 100. Three kinds of instabilities are found: oblique wave, streamwise wave, and spanwise stationary mode, whose properties are all significantly affected by elasticity. For the first, the critical Marangoni number increases with elasticity. For the second, the work done by perturbation stress fluctuates in vertical direction. The last becomes the preferred mode when the elasticity is high enough and its perturbation energy comes from the Marangoni force caused by perturbation temperature while dissipates by perturbation stress. Their mechanisms are discussed and the comparisons are made with Newtonian fluid. (C) 2016 AIP Publishing LLC. [http://dx.doi.org/10.1063/1.4943971]
\end{abstract}

\section{INTRODUCTION}

Thermocapillary convection refers to the fluid motion driven by temperature-dependent surface tension, where the fluid layer is subjected to a horizontal temperature gradient on the surface. It has always received a wide range of interest in the last few decades for its fundamental and practical importance. In many technological fields, it has an important or even a dominant effect, including crystal growth, ${ }^{1}$ film rupture, ${ }^{2}$ thin-film coating, ${ }^{3}$ fusion welding,${ }^{4-6}$ and combustion. ${ }^{7}$ There have been plenty of theoretical and experimental studies of thermocapillary flows, which have been reviewed by Davis ${ }^{8}$ and Schatz and Neitzel. ${ }^{2}$

In many industrial applications, there are some physical systems involving non-uniform heating on the surface of polymer liquid, such as plastic welding ${ }^{9-11}$ and film drying. ${ }^{12-14}$ There have been plenty of works on the surface tension of polymer liquid, which has been reviewed by Dee and Sauer. ${ }^{15}$ It has been observed that the surface tension for polymer molecules is also linear with the temperature over limited temperature domains. This may lead to thermocapillary effect and makes a great influence on the quality of products in materials processing, for example, the local thinning ${ }^{16}$ and surface tension driven defects in liquid film. ${ }^{17}$ However, the polymer liquids are often viscoelastic fluids, whose flow properties are affected by elasticity significantly. Viscoelastic models should be used in the study of thermocapillary flows for polymer liquids.

The upper-convected Maxwell (UCM) fluid and Oldroyd-B fluid are two non-linear viscoelastic models which are mainly used in theoretical studies. Comparing to linear models, they can describe the normal stress effects in shear flows more properly. Their constitutive equations can be derived from kinetic theory where the spring-dumbbell model is used to represent polymer molecules. ${ }^{18-20}$ The Oldroyd-B fluid can be obtained for a dilute suspension of spring-dumbbells in a Newtonian solvent. When the polymer contribution to the fluid viscosity is far larger than that of the solvent, the UCM fluid is derived. ${ }^{21}$

In the theoretical studies, thermocapillary liquid layer is a convenient model, where the flow above an infinite flat plane is subjected to a horizontal temperature gradient on the surface. For Newtonian fluids, there are two types of instabilities, which are stationary longitudinal rolls and unstable hydrothermal waves, respectively. ${ }^{22}$ For viscoelastic fluids, a few papers have been devoted to the study of Benard-Marangoni convection, where a vertical temperature gradient is imposed to the fluid layer. ${ }^{23-29}$ Concerning the liquid layer with a horizontal temperature gradient, we are only 
aware of the work given by Kaloni and Lou, ${ }^{30}$ where the influence of thermocapillary forces on the buoyancy-driven viscoelastic fluid layer has been demonstrated for Oldroyd-B fluid with Prandtl numbers 10 and 100.

Our previous paper has examined the linear stability of thermocapillary liquid layers for UCM fluid. ${ }^{31}$ Elastic instability is found for oblique waves. In contrast to Newtonian fluids, ${ }^{22}$ unstable modes are detected at low Reynolds numbers when the wave number is high enough. The wave speed and propagation direction of the most unstable mode keep constant for different basic flows. It suggests that the stabilities of viscoelastic thermocapillary flows are very different from those for Newtonian fluids. However, in the present work, we find that the elastic waves decay rapidly with the increasing of the solvent viscosity in Oldroyd-B fluid. This makes its instability quite different from that for the UCM fluid.

The purpose of this paper is to generalize the study of the instability of viscoelastic thermocapillary liquid layers to Oldroyd-B fluid. Buoyancy is not considered in our work. The flow includes linear flow and return flow. The results are presented for Prandtl numbers 0.02 and 100 .

This paper is organized as follows. In Section II, the mathematical formulations are introduced and the dimensionless governing equations are derived. Then in Section III, the numerical results are presented. The most unstable modes at different elasticities are studied and the critical Marangoni numbers are obtained. The critical wave speed, wave number, and wave propagation angle are derived. The perturbation flow field is plotted and the energy analysis is proposed. Later, the mechanisms are discussed in Section IV. Finally, the main results are summarized in Section V.

\section{PROBLEM FORMULATION}

We use the same model and coordinate as the work by Smith and Davis, ${ }^{22}$ where a fluid layer is above an infinite rigid plane and a temperature gradient is imposed on its free surface (see Fig. 1). $\mathrm{x}, \mathrm{y}, \mathrm{z}$ are the streamwise, spanwise, and wall-normal direction, respectively. The dimensionless parameters are defined as follows: $R$ is the Reynolds number $R=\frac{\rho U d}{\mu}$, where $\rho, U, d, \mu$ are the fluid density, characteristic velocity, the depth of the fluid layer, and viscosity, respectively. Here, $U=b \gamma d / \mu, b$ is the temperature gradient on the surface, and $\gamma$ is the negative rate of change of surface tension with temperature. $M a$ is the Marangoni number defined as $M a=b \gamma d^{2} / \mu \chi, \chi$ is the thermal diffusivity. $M a$ and $R$ have the relation $M a=R \cdot P r$, where $P r$ is the Prandtl number. These definitions are the same as those in Ref. 22.

\section{A. Governing equations}

The length, velocity, and temperature difference scales are set as $d, U$, and $b d$, respectively. Thus, the dimensionless governing equations can be derived as follows by dividing these scales to the dimensional variables, which are the continuity equation, the momentum equation, and the energy equation, respectively,

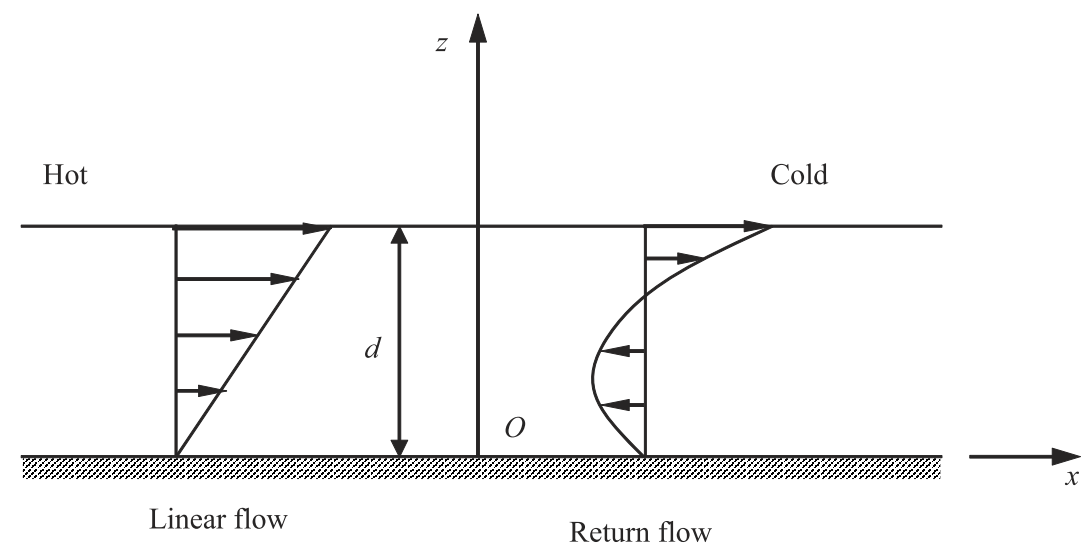

FIG. 1. The schematic of thermocapillary liquid layers. 


$$
\begin{aligned}
\nabla \cdot \mathbf{u} & =0, \\
R\left(\frac{\partial \mathbf{u}}{\partial t}+\mathbf{u} \cdot \nabla \mathbf{u}\right) & =-\nabla p+\nabla \cdot \mathbf{Q}, \\
\frac{\partial T}{\partial t}+\mathbf{u} \cdot \nabla T & =\frac{1}{M a} \nabla^{2} T .
\end{aligned}
$$

Here $\mathbf{u}, p, T$ stand for the velocity field, pressure, and temperature, respectively. $\mathbf{Q}$ is the stress tensor. For an Oldroyd-B fluid, its dimensionless constitutive relation is given by the equation ${ }^{32}$

$$
\left(1+\lambda \frac{\delta}{\delta t}\right) \mathbf{Q}=\left(1+\eta \frac{\delta}{\delta t}\right) \mathbf{S}
$$

It can be derived by dividing $\mu U / d$ to the dimensional form. Here $\lambda$ is the Weissenberg number, which has $\lambda=\frac{\mu}{G} \frac{U}{d}, G$ is the elastic modulus. $\eta / \lambda$ represents the ratio of solvent to the total viscosity, ${ }^{33} \mathbf{S}$ is the strain-rate tensor with the form $\mathbf{S}=\nabla \mathbf{u}+\mathbf{u} \nabla$, and $\frac{\delta}{\delta t}$ is the upper convected derivative with the expression

$$
\frac{\delta \mathbf{Q}}{\delta t}=\frac{\partial \mathbf{Q}}{\partial t}+\mathbf{u} \cdot \nabla \mathbf{Q}-(\mathbf{u} \nabla) \cdot \mathbf{Q}-\mathbf{Q} \cdot(\nabla \mathbf{u})
$$

It should be noted that Oldroyd-B fluid recovers UCM fluid when $\eta=0$ and Newtonian fluid when $\lambda=\eta=0$. We use elastic number $\varepsilon=\lambda / R$ in the following computation as it only depends on the properties of the fluid and the flow geometry. ${ }^{33}$

The boundary conditions are set as the following:

$$
\begin{gathered}
z=0: \quad \mathbf{u}=(u, v, w)=0, \frac{\partial T}{\partial z}=0, \\
z=1: \quad Q_{13}+\frac{\partial T}{\partial x}=0, Q_{23}+\frac{\partial T}{\partial y}=0, w=0, \frac{\partial T}{\partial z}=0 .
\end{gathered}
$$

Here, the heat flux through the plane and surface are both zero, the stress on the surface is caused by thermocapillary effect.

We consider two kinds of basic flows as the work by Smith and Davis, ${ }^{22}$ which includes linear flow and return flow (see Fig. 1). Both of them are plane shear flows on an infinite rigid plane, and the fluid is set in motion by the temperature gradient on the free surface. The former has homogeneous velocity gradient, while the latter, which is a sum of plane Couette and plane Poiseuille flow, has zero mass flux through any vertical section. The temperature field is linear in $\mathrm{x}$ as imposed plus a distribution in $\mathrm{z}$, which maintains a balance between conduction and convection. The solutions of the basic flow for Oldroyd-B fluid have the same form as those for Newtonian fluid, ${ }^{22}$ except the normal stress in streamwise direction,

$$
\begin{gathered}
u_{0}=u_{0}^{\prime}(1) z+\frac{1}{2} u_{0}^{\prime \prime}(1)\left(z^{2}-2 z\right), v_{0}=w_{0}=0, \\
T_{0}=-u_{0}^{\prime}(1) x+M a \cdot u_{0}^{\prime}(1)\left\{\frac{1}{24} u_{0}^{\prime \prime}(1)\left(1-z^{4}\right)+\frac{1}{6}\left[u_{0}^{\prime}(1)-u_{0}^{\prime \prime}(1)\right]\left(1-z^{3}\right)\right\}, \\
\mathbf{S}_{0}=u_{0}^{\prime}(z)\left[\begin{array}{ccc}
0 & 0 & 1 \\
0 & 0 & 0 \\
1 & 0 & 0
\end{array}\right], \mathbf{Q}_{0}=u_{0}^{\prime}(z)\left[\begin{array}{ccc}
2(\lambda-\eta) u_{0}^{\prime}(z) & 0 & 1 \\
0 & 0 & 0 \\
1 & 0 & 0
\end{array}\right] .
\end{gathered}
$$

For linear flow, we have $u_{0}^{\prime}(1)=1, u_{0}^{\prime \prime}(1)=0$ while for return flow $u_{0}^{\prime}(1)=1, u_{0}^{\prime \prime}(1)=\frac{3}{2} \cdot u_{0}^{\prime}(z)$ stands for the derivative of $z$.

\section{B. Perturbation equations}

A small perturbation in the normal mode form is added to the basic flow,

$$
\begin{gathered}
\mathbf{u}=\mathbf{u}_{0}+(\widehat{u}, \widehat{v}, \widehat{w}) \exp [\sigma t+\mathrm{i}(\alpha x+\beta y)], \\
T=T_{0}+\widehat{T} \exp [\sigma t+\mathrm{i}(\alpha x+\beta y)],
\end{gathered}
$$




$$
\begin{gathered}
P=P_{0}+\widehat{P} \exp [\sigma t+\mathrm{i}(\alpha x+\beta y)], \\
\mathbf{Q}=\mathbf{Q}_{0}+\left[\begin{array}{lll}
e & r & f \\
r & q & g \\
f & g & h
\end{array}\right] \exp [\sigma t+\mathrm{i}(\alpha x+\beta y)],
\end{gathered}
$$

in which $\sigma=\sigma_{r}+\mathrm{i} \sigma_{i}$. The wave number and direction of wave propagation are defined as $k=$ $\sqrt{\alpha^{2}+\beta^{2}}, \phi=\tan ^{-1}(\beta / \alpha)$, respectively. Due to symmetry, we shall confine ourselves to the case of $\phi \in\left[0^{\circ}, 180^{\circ}\right]$. Hereafter, the variables without subscript 0 stand for the perturbation. Substituting (2.9a)-(2.9d) into governing equations, the linearized perturbation equations can be derived,

$$
\begin{gathered}
i \alpha \widehat{u}+i \beta \hat{v}+\widehat{w}^{\prime}=0, \\
R\left(\sigma \widehat{u}+\widehat{w} u_{0}^{\prime}+u_{0} i \alpha \widehat{u}\right)=-i \alpha \widehat{p}+i \alpha \hat{e}+i \beta \widehat{r}+\widehat{f}^{\prime}, \\
R\left(\sigma \widehat{v}+u_{0} i \alpha \widehat{v}\right)=-i \beta \widehat{p}+i \alpha \widehat{r}+i \beta \widehat{q}+\widehat{g}^{\prime}, \\
R\left(\sigma \widehat{w}+u_{0} i \alpha \widehat{w}\right)=-\widehat{p}^{\prime}+i \alpha \widehat{f}+i \beta \widehat{g}+\widehat{h}^{\prime}, \\
M a\left(\widehat{u} \frac{\partial T_{0}}{\partial x}+\widehat{w} \frac{\partial T_{0}}{\partial z}+u_{0} i \alpha \widehat{T}\right)+\left(\alpha^{2}+\beta^{2}\right) \widehat{T}-\widehat{T}^{\prime \prime}=-\sigma M a \widehat{T},
\end{gathered}
$$

$\left(1+\lambda\left(u_{0} i \alpha\right)\right) e-\left(1+\eta\left(u_{0} i \alpha\right)\right) 2 i \alpha \widehat{u}-\lambda u_{0}^{\prime} 2 f+\lambda\left(\widehat{w} u_{0}^{\prime \prime} \cdot u_{0}^{\prime} 4(\lambda-\eta)-u_{0}^{\prime}\left(4(\lambda-\eta) u_{0}^{\prime} i \alpha \widehat{u}\right.\right.$ $\left.\left.+2 \widehat{u}^{\prime}\right)\right)+\eta u_{0}^{\prime}\left(2 \widehat{u}^{\prime}+2 i \alpha \widehat{w}\right)+\eta u_{0}^{\prime} 2 \widehat{u}^{\prime}=\sigma(\eta 2 i \alpha \widehat{u}-\lambda e)$,

$$
\begin{aligned}
& \left(1+\lambda\left(u_{0} i \alpha\right)\right) r-\left(1+\eta\left(u_{0} i \alpha\right)\right)(i \alpha \widehat{v}+i \beta \widehat{u})-\lambda u_{0}^{\prime} g+\lambda\left(-u_{0}^{\prime}\left(2(\lambda-\eta) u_{0}^{\prime} i \alpha \widehat{v}+\widehat{v}^{\prime}\right)\right) \\
& \quad+\eta u_{0}^{\prime}\left(i \beta \widehat{w}+\widehat{v}^{\prime}\right)+\eta u_{0}^{\prime} \widehat{v}^{\prime}=\sigma(\eta(i \alpha \widehat{v}+i \beta \widehat{u})-\lambda r), \\
& \left(1+\lambda\left(u_{0} i \alpha\right)\right) f-\left(1+\eta\left(u_{0} i \alpha\right)\right)\left(\widehat{u}^{\prime}+i \alpha \widehat{w}\right)-\lambda u_{0}^{\prime} h+\lambda\left(\widehat{w} u_{0}^{\prime \prime}-u_{0}^{\prime}\left(2(\lambda-\eta) u_{0}^{\prime} i \alpha \widehat{w}\right.\right. \\
& \left.\left.\quad+\widehat{w}^{\prime}+i \alpha \widehat{u}\right)\right)+\eta u_{0}^{\prime}\left(2 \widehat{w}^{\prime}\right)-\eta\left(\widehat{w} u_{0}^{\prime \prime}-u_{0}^{\prime}\left(\widehat{w}^{\prime}+i \alpha \widehat{u}\right)\right)=\sigma\left(\eta\left(\widehat{u}^{\prime}+i \alpha \widehat{w}\right)-\lambda f\right),
\end{aligned}
$$

$$
\left(1+\lambda\left(u_{0} i \alpha\right)\right) q-\left(1+\eta\left(u_{0} i \alpha\right)\right)(2 i \beta \widehat{v})=\sigma(\eta(2 i \beta \widehat{v})-\lambda q),
$$

$\left(1+\lambda\left(u_{0} i \alpha\right)\right) g-\left(1+\eta\left(u_{0} i \alpha\right)\right)\left(i \beta \widehat{w}+\widehat{v}^{\prime}\right)-\lambda u_{0}^{\prime} i \alpha \widehat{v}+\eta u_{0}^{\prime} i \alpha \widehat{v}=\sigma\left(\eta\left(i \beta \widehat{w}+\widehat{v}^{\prime}\right)-\lambda g\right)$,

$\left(1+\lambda\left(u_{0} i \alpha\right)\right) h-\left(1+\eta\left(u_{0} i \alpha\right)\right)\left(2 \widehat{w}^{\prime}\right)-\lambda u_{0}^{\prime} 2 i \alpha \widehat{w}+\eta u_{0}^{\prime} 2 i \alpha \widehat{w}=\sigma\left(\eta\left(2 \widehat{w}^{\prime}\right)-\lambda h\right)$.

The boundary conditions of perturbation flow are

$$
\begin{gathered}
\widehat{u}=\widehat{v}=\widehat{w}=\frac{\partial \widehat{T}}{\partial z}=0, z=0, \\
f+i \alpha \widehat{T}=0, g+i \beta \widehat{T}=0, \widehat{w}=0, \frac{\partial \widehat{T}}{\partial z}=0, z=1 .
\end{gathered}
$$

Chebyshev collocation method is used to solve the generalized eigenvalue problem and more than 80 Chebyshev modes are applied to ensure the accuracy.

\section{Code validation}

The code we used is based on that for UCM fluid. The only differences of the codes for these two fluids are the constitutive equations and the stress of basic flow. It is known that Oldroyd-B fluid tends to Newtonian fluid when $\eta / \lambda \rightarrow 1$, which can also be seen from the constitutive equation. In order to validate our code, we compute the eigenvalues of Oldroyd-B fluid with $\eta / \lambda \rightarrow 1$ and compare the results with Newtonian fluid. The comparison of most unstable eigenvalues between them is displayed in Table I, which are exactly the same with each other.

We compare the critical Marangoni number and propagation angle of hydrothermal waves determined by linear stability analysis for an infinite liquid layer with the numerical simulation results for annular pools in Table II. It can be seen that the propagation angle is in good agreement with the results of $\mathrm{Li}$ et al., ${ }^{34,35}$ while the Marangoni number for the appearance of hydrothermal wave is different in different geometries. 
TABLE I. A comparison of most unstable eigenvalues for Newtonian fluid and Oldroyd-B fluid at $\operatorname{Pr}=0.02, M a=6.15, k=0.39, \phi=86.3^{\circ}$ (linear flow).

\begin{tabular}{lc}
\hline \hline Newtonian fluid $(\lambda=\eta=0)$ & Oldroyd-B fluid at $\varepsilon=0.01, \eta / \lambda \rightarrow 1$ \\
\hline $0.000026+0.031995 \mathrm{i}$ & $0.000026+0.031995 \mathrm{i}$ \\
$-0.000001-0.012584 \mathrm{i}$ & $-0.000001-0.012584 \mathrm{i}$ \\
$-0.000613-0.062832 \mathrm{i}$ & $-0.000613-0.062832 \mathrm{i}$ \\
$-0.065904-0.016560 \mathrm{i}$ & $-0.065904-0.016560 \mathrm{i}$ \\
$-0.108399-0.011974 \mathrm{i}$ & $-0.108399-0.011974 \mathrm{i}$ \\
\hline \hline
\end{tabular}

\section{NUMERICAL RESULTS}

In our previous work on the thermocapillary liquid layers for UCM fluid, unstable oblique waves are detected at low Reynolds numbers. ${ }^{31}$ Their wave speeds have $c \propto \frac{1}{\sqrt{R \lambda}}$ when other parameters are fixed. Thus, the dimensional form of the wave speeds has $C=c U \propto \frac{U}{\sqrt{R \lambda}}=\sqrt{\frac{G}{\rho}}$, which is obviously an elastic wave speed. In Table III, the case for $\eta / \lambda=0$ corresponds to UCM fluid, unstable wave exists when $R=M a / P r=0.03$.

The elastic wave is the most unstable mode for UCM fluid. However, it decays rapidly with the increasing of the solvent viscosity in Oldroyd-B fluid (see Table III). It is observed that when $\eta / \lambda$ increases, the frequency $\sigma_{i}$ nearly keeps the same while the growth rate $\sigma_{r}$ decreases significantly. This is conceivable. Comparing with the UCM model, the Oldroyd-B model adds a Newtonian component, which can make viscous dissipation at low Reynolds numbers. When $\eta / \lambda$ is not very small, the most unstable mode changes to other kinds of mode (for example, the spanwise stationary mode for $\eta / \lambda \geq 0.1$ in Fig. 4). Thus, the instability of Oldroyd-B fluid is quite different from that for UCM fluid.

In Table IV, we list the eigenvalues for Oldroyd-B fluid at two elastic numbers. It can be seen that there are two kinds of modes: the first five eigenvalues nearly keep constant while the last two eigenvalues change a lot with $\varepsilon$. The wave speeds of the second kind modes have $c=\frac{\left|\sigma_{\mathrm{i}}\right|}{k} \propto \frac{1}{\sqrt{\varepsilon}}$. (As $\varepsilon=\lambda / R$, we have $c \propto \frac{1}{\sqrt{R \lambda}}$ for fixed $R$.) When $\varepsilon \rightarrow 0$, the first kind still exists while the second kind decays. Thus we can infer that the latter is mainly caused by elasticity while the former is not. As UCM fluid can be seen as the special case of Oldroyd-B fluid for $\eta=0$, the existence of elastic mode in Oldroyd-B fluid is conceivable. There is also a critical wave number for Oldroyd-B fluid, which is similar to that for UCM fluid. In Fig. 2, the growth rate of elastic mode for Oldroyd-B fluid increases with $k$ and exceeds zero when $k$ reaches the critical value. However, the elastic mode for Oldroyd-B fluid decays rapidly with $\eta$ and its critical wave number increases dramatically. When $\eta / \lambda \geq 0.005$, we cannot find an unstable elastic mode for $k \leq 10^{5}$. So later, we do not consider the elastic mode and restrict our tension to the first kind of modes for $\eta / \lambda \geq 0.1$. The results for linear flow and return flow will be discussed separately.

The parameters $\operatorname{Pr}, \varepsilon, \lambda / \eta$ only depend on the fluid properties and flow geometry. ${ }^{33}$ When they are fixed, we compute the eigenvalues at different wave numbers and propagation directions to find the preferred mode, which reaches neutral stability with the smallest Marangoni number $M a_{c}$. We call it the critical Marangoni number in the following.

TABLE II. The critical Marangoni number and propagation angle for the appearance of hydrothermal waves at $P r=0.01$. Comparison with reference values by numerical simulations.

\begin{tabular}{|c|c|c|c|c|}
\hline & \multicolumn{2}{|c|}{ Numerical simulation } & \multicolumn{2}{|c|}{$\begin{array}{l}\text { Linear stability analysis for an } \\
\text { infinite liquid layer }\end{array}$} \\
\hline & Li et al. (annular pool) $)^{34}$ & $\begin{array}{l}\text { Li et al. (pool with } \mathrm{Cz} \\
\text { configuration) } \\
\text { ch }^{35}\end{array}$ & Smith and Davis ${ }^{22}$ & Present work \\
\hline$M a$ & 11.94 & 24.40 & 6.29 & 6.29 \\
\hline Angle of propagation & 78 & 78 & 77 & 77 \\
\hline
\end{tabular}


TABLE III. The most unstable eigenvalues of linear flow for Oldroyd-B fluid at $\operatorname{Pr}=100, M a=3, k=10, \phi=55^{\circ}, \varepsilon=2$.

\begin{tabular}{lr}
\hline \hline$\eta / \lambda$ & $\sigma$ \\
\hline 0 & $2.694084-253.071634 \mathrm{i}$ \\
0.001 & $0.818839-253.002646 \mathrm{i}$ \\
0.002 & $-1.055771-252.919687 \mathrm{i}$ \\
0.005 & $-6.675798-252.586828 \mathrm{i}$ \\
\hline \hline
\end{tabular}

\section{A. Linear flow}

The neutral curves for linear flow with $\operatorname{Pr}=100, \phi=90^{\circ}$ are displayed in Fig. 3 with various values of $\varepsilon$ and $\eta / \lambda$. The Marangoni number of neutral mode always increases with $k$ and reaches the minimum for $k \rightarrow 0$.

The variation of critical Marangoni number $M a_{c}$ with $\varepsilon$ for linear flow at $\operatorname{Pr}=100$ is displayed in Fig. 4. The computation shows that the preferred mode is the spanwise stationary mode $\left(\phi=90^{\circ}\right)$, which is the same as the case for Newtonian fluid (the stationary longitudinal rolls ${ }^{22}$ ).

$M a_{c}$ increases with $\varepsilon$ and decrease with $\eta / \lambda$. Physically, these suggest that the elasticity can destabilize the spanwise stationary mode while the solvent viscosity is stabilizing. As the increasing of $\eta / \lambda$ always makes the flow stability approach to that for Newtonian fluid, for simplicity, we shall confine ourselves to the case for $\eta / \lambda=0.1$ and discuss the impact of $\varepsilon$ for the flow stability in the following study.

The variation of critical wave number $k_{c}$ for spanwise stationary mode with $\varepsilon$ for linear flow at $\operatorname{Pr}=100, \eta / \lambda=0.1$ is displayed in Fig. 5. It is observed that $k_{c}$ decreases with $\varepsilon$ rapidly, and tends to 0 for large $\varepsilon$.

Similar results hold for the cases we studied in the following for $\operatorname{Pr}=0.02$ and return flow. The spanwise stationary mode becomes the preferred mode when $\varepsilon$ is high enough, meanwhile its critical wave number tends to 0 .

The variation of $M a_{c}$ with $\varepsilon$ for linear flow at $\operatorname{Pr}=0.02, \eta / \lambda=0.1$ is displayed in Fig. 6 . The preferred mode changes with the increasing of $\varepsilon$. For $\varepsilon<0.009$, the preferred mode is the oblique wave $\left(\phi \neq 0^{\circ}, 90^{\circ}\right), M a_{c}$ increases slightly with $\varepsilon$; for $0.009<\varepsilon<0.07$, the preferred mode is the streamwise wave $\left(\phi=0^{\circ}\right)$; for $\varepsilon>0.07$, it becomes the spanwise stationary mode $\left(\phi=90^{\circ}\right) . M a_{c}$ for the last two modes decreases with $\varepsilon$ significantly.

Fig. 7 shows the wave number $k_{c}$ corresponding to the oblique wave and streamwise wave in Fig. 6. For the former, $k_{c}$ is smaller and increases slightly with $\varepsilon$ while for the latter, $k_{c}$ is larger and decreases with $\varepsilon$ significantly. For the spanwise stationary mode, $k_{c} \rightarrow 0$.

In Fig. 8, we plot the wave propagation angle $\phi_{c}$ corresponding to the oblique wave in Fig. 6 . When $\varepsilon$ is small, $\phi_{c}$ decreases with $\varepsilon$ and changes from $\phi_{c}>90^{\circ}$ to $\phi_{c}<90^{\circ}$ at $\varepsilon \approx 0.003$, which suggests that the oblique wave changes from upstream to downstream. When $\varepsilon>0.035, \phi_{c}$ starts to increase with $\varepsilon$.

The wave speed corresponding to the oblique wave and streamwise wave in Fig. 6 is displayed in Fig. 9. The former increases with $\varepsilon$ to some extent and then decreases slightly while the latter

TABLE IV. The eigenvalues $\sigma$ of linear flow for Oldroyd-B fluid at two elastic numbers at $\operatorname{Pr}=100, M a=1.71, k=3, \phi=55^{\circ}, \eta / \lambda=0.1$.

\begin{tabular}{cc}
\hline \hline$\varepsilon=0.3$ & \multicolumn{1}{c}{$\varepsilon=0.5$} \\
\hline$-5.279624-0.866043 \mathrm{i}$ & $-5.279639-0.865904 \mathrm{i}$ \\
$-10.963913-0.927956 \mathrm{i}$ & $-10.964437-0.925270 \mathrm{i}$ \\
$-28.342864-0.920475 \mathrm{i}$ & $-28.342548-0.914101 \mathrm{i}$ \\
$-57.214140-0.878765 \mathrm{i}$ & $-57.211826-0.873992 \mathrm{i}$ \\
$-97.614137-0.872966$ & $-97.610748-0.864717 \mathrm{i}$ \\
$-130.997657+335.782333 \mathrm{i}$ & $-92.012657+263.306667 \mathrm{i}$ \\
$-130.997654-338.200447 \mathrm{i}$ & $-92.012646-265.724781 \mathrm{i}$ \\
\hline \hline
\end{tabular}




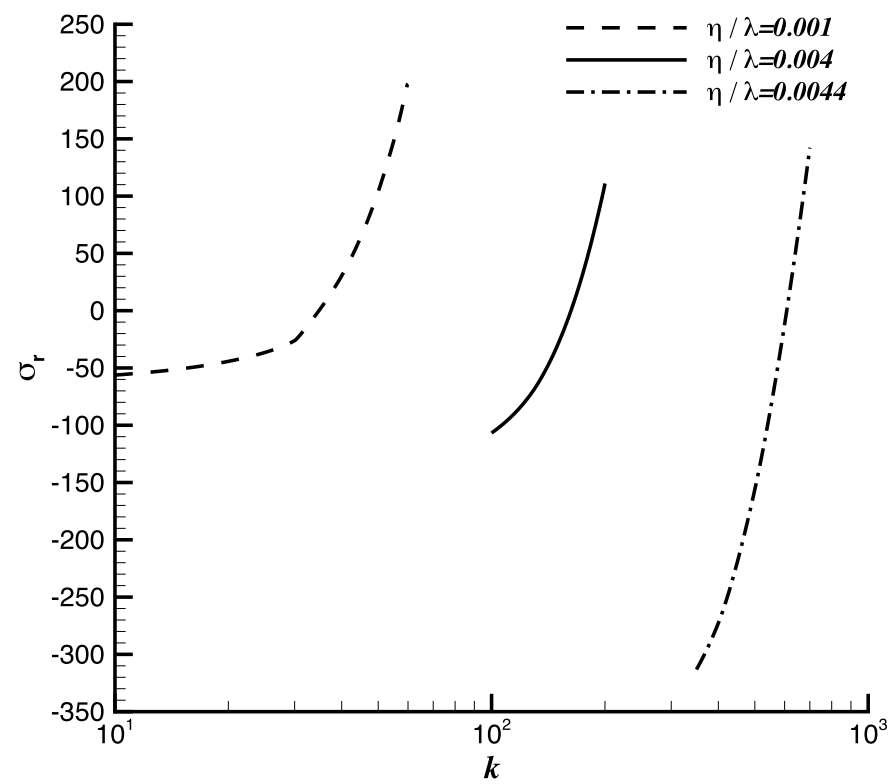

FIG. 2. The variation of the growth rate for elastic mode with the wave number for linear flow at $\operatorname{Pr}=100, \operatorname{Ma}=1.71$, $\theta=55^{\circ}, \varepsilon=0.5$.

is always decreasing. However, the former is smaller than the latter for each $\varepsilon$. The wave speed of spanwise stationary mode is zero.

\section{B. Return flow}

The variation of $M a_{c}$ with $\varepsilon$ for return flow is displayed in Fig. 10 at $\operatorname{Pr}=100, \eta / \lambda=0.1$. The preferred mode also consists of three kinds. For $\varepsilon<0.09$, the preferred mode is the upstream oblique wave $\left(\phi>90^{\circ}\right), M a_{c}$ increases with $\varepsilon$ continuously; for $0.09<\varepsilon<0.3$, the preferred mode is the downstream oblique wave $\left(\phi<90^{\circ}\right)$; for $\varepsilon>0.3$, it becomes the spanwise stationary mode. $M a_{c}$ for the last two modes decreases with $\varepsilon$ swiftly.

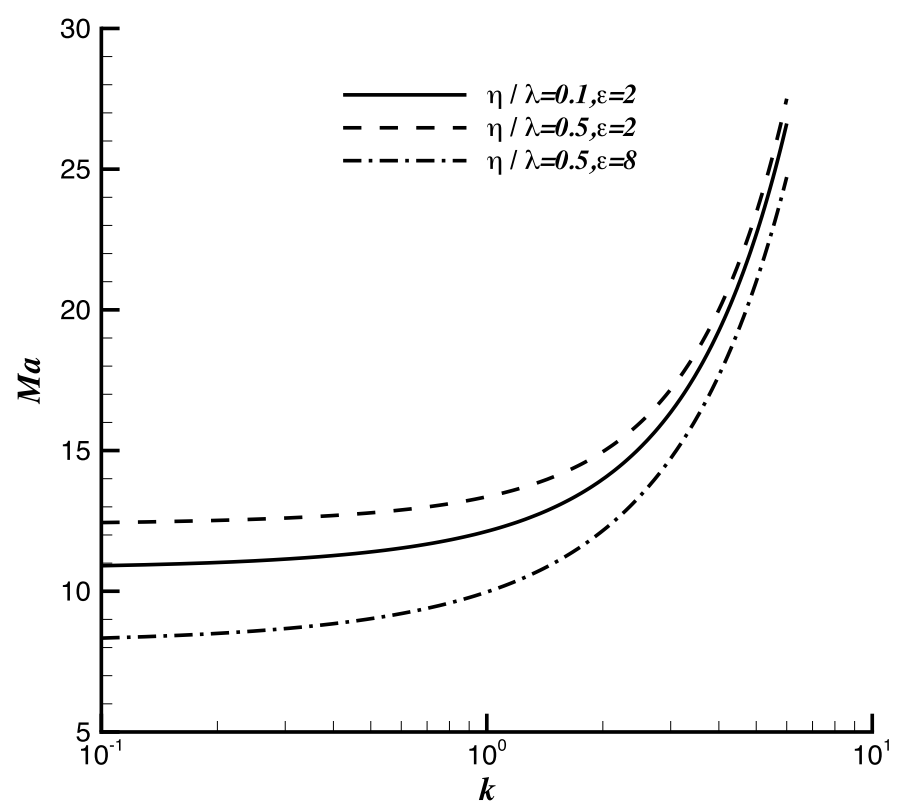

FIG. 3. The neutral curves for linear flow with various values of $\varepsilon$ and $\eta / \lambda$ at $\operatorname{Pr}=100, \phi=90^{\circ}$. 


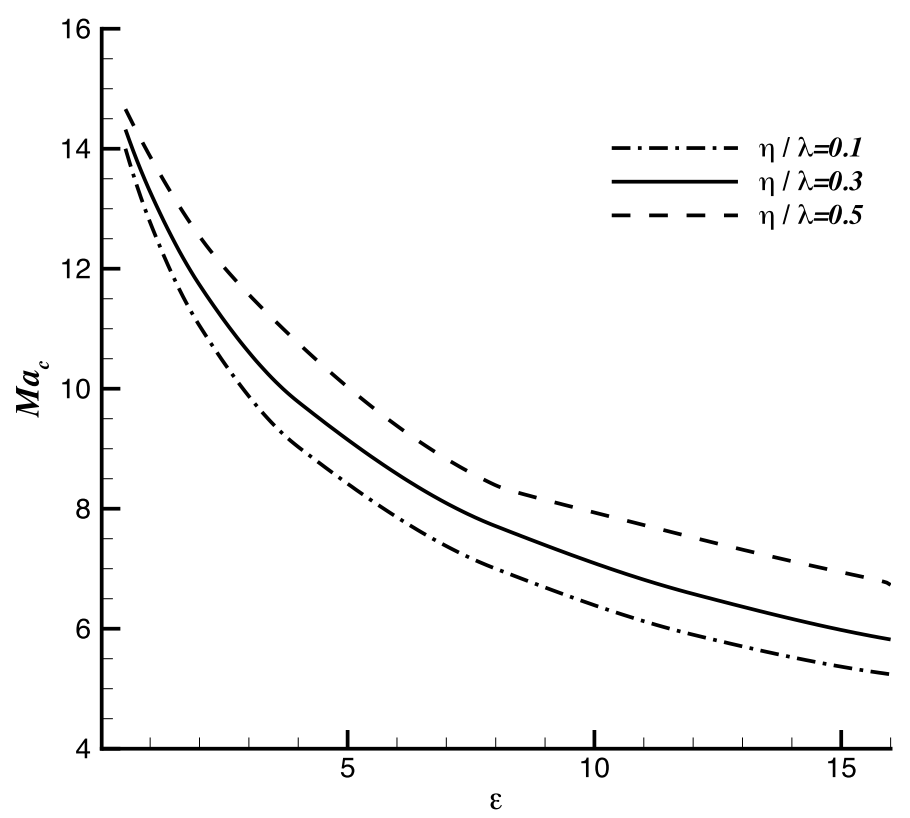

FIG. 4. The variation of critical Marangoni number $M a_{c}$ with $\varepsilon$ for linear flow at $\operatorname{Pr}=100$. The preferred mode is the spanwise stationary mode $\left(\phi=90^{\circ}\right)$.

In Figs. 11-13, we plot the wave number, wave propagation angle, and wave speed corresponding to the upstream and downstream oblique waves in Fig. 10. Both of their wave numbers and propagation angles decrease with $\varepsilon$. For the wave speed, the one for upstream oblique wave increases slightly with $\varepsilon$ while the one for downstream oblique wave decreases obviously.

Although the oblique wave in Fig. 6 can also propagate upstream and downstream (see Fig. 8), its wave number, wave speed, and propagation angle change with $\varepsilon$ continuously. In contrast, the upstream oblique wave in Fig. 10 cannot transit to the downstream oblique wave continuously, which can be seen in Figs. 11-13. So they are different modes.

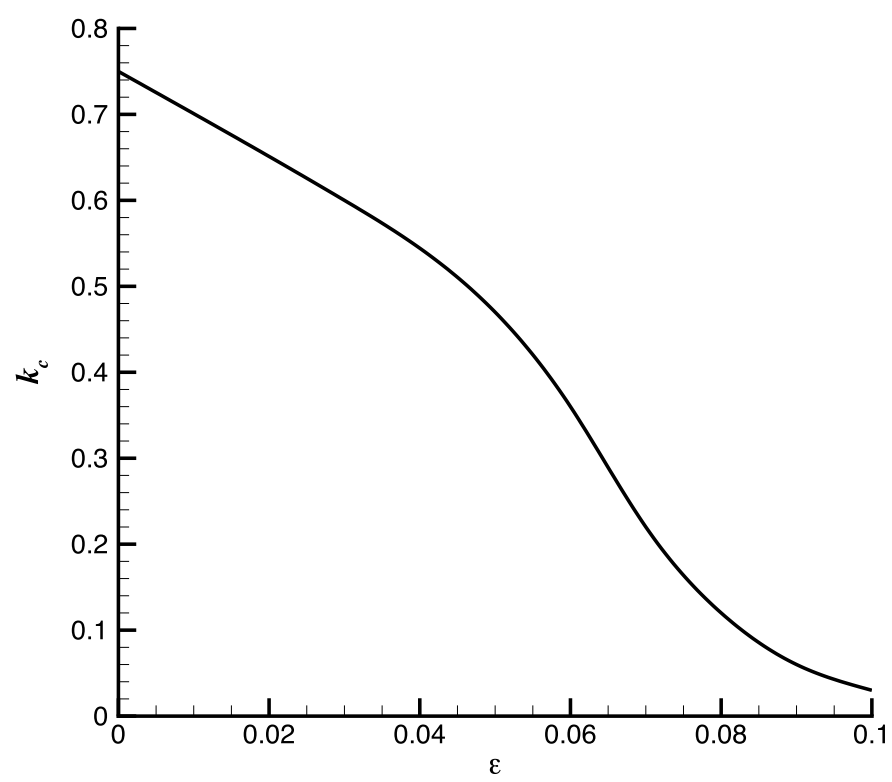

FIG. 5. The variation of critical wave number $k_{c}$ for spanwise stationary mode with $\varepsilon$ for linear flow at $\operatorname{Pr}=100, \eta / \lambda=0.1$. 


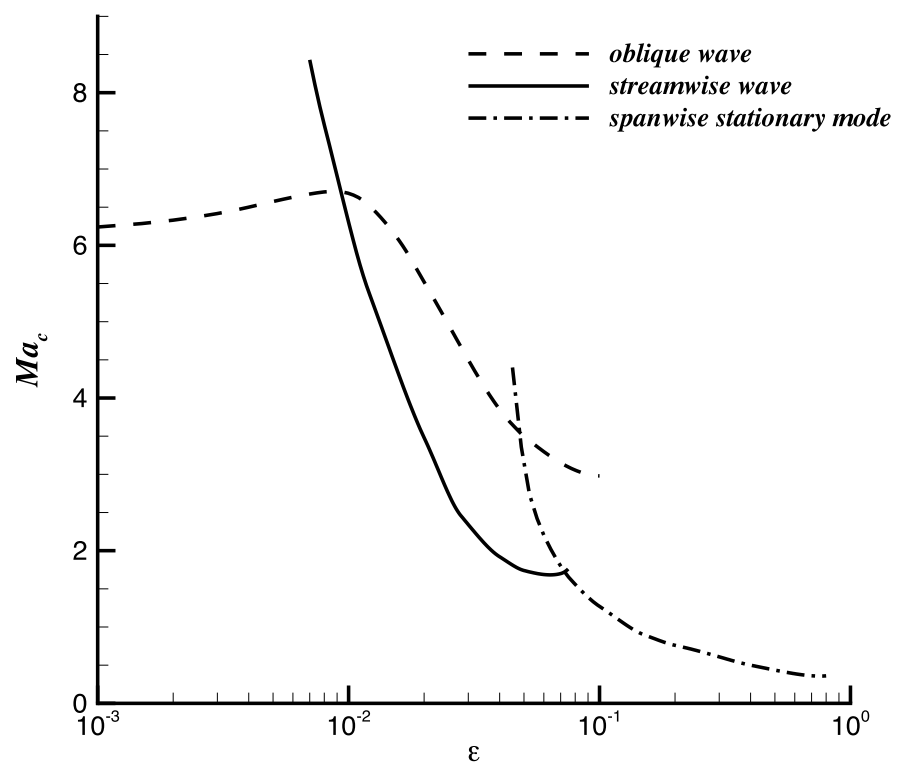

FIG. 6. The variation of $M a_{c}$ with $\varepsilon$ for linear flow at $\operatorname{Pr}=0.02, \eta / \lambda=0.1$.

The variation of $M a_{c}$ with $\varepsilon$ for return flow is displayed in Fig. 14 at $\operatorname{Pr}=0.02, \eta / \lambda=0.1$. To some extent, it is similar to the case for linear flow at $\mathrm{Pr}=0.02$ in Fig. 6 . The preferred modes are the oblique wave for $\varepsilon<0.006$, the streamwise wave for $0.006<\varepsilon<0.025$, and the spanwise stationary mode for $\varepsilon>0.025$. For the first mode, $M a_{c}$ increases slightly with $\varepsilon$ while for the last two modes, it decreases with $\varepsilon$ significantly. The corresponding wave number, wave propagation angle, and wave speed for these modes are displayed in Figs. 15-17. Most of their variations with $\varepsilon$ are similar to those for linear flow in Figs. 7-9. The only exception is the wave speed for the oblique wave, which decreases with $\varepsilon$.

It is worth noting that there is no stationary instability in return flow for the Newtonian fluid. ${ }^{22}$ In contrast, the spanwise stationary mode is the preferred mode for Oldroyd-B fluid in both linear flow and return flow when $\varepsilon$ is high enough, which suggests a different mechanism from that in Newtonian fluid. We will give an explanation in Section IV.

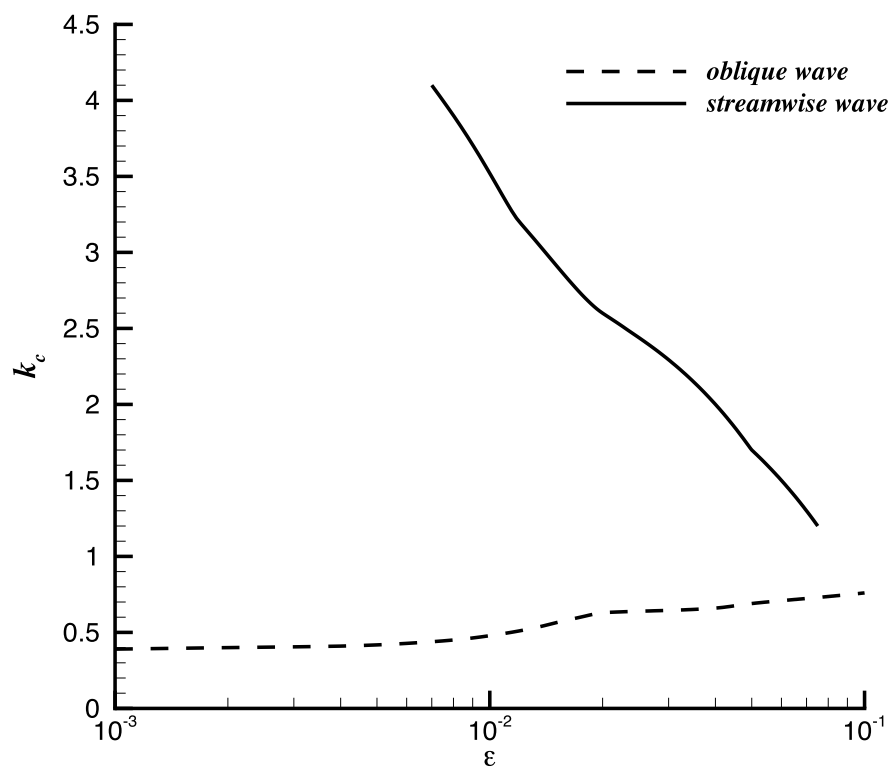

FIG. 7. The wave number corresponding to the oblique wave and streamwise wave in Fig. 6. 


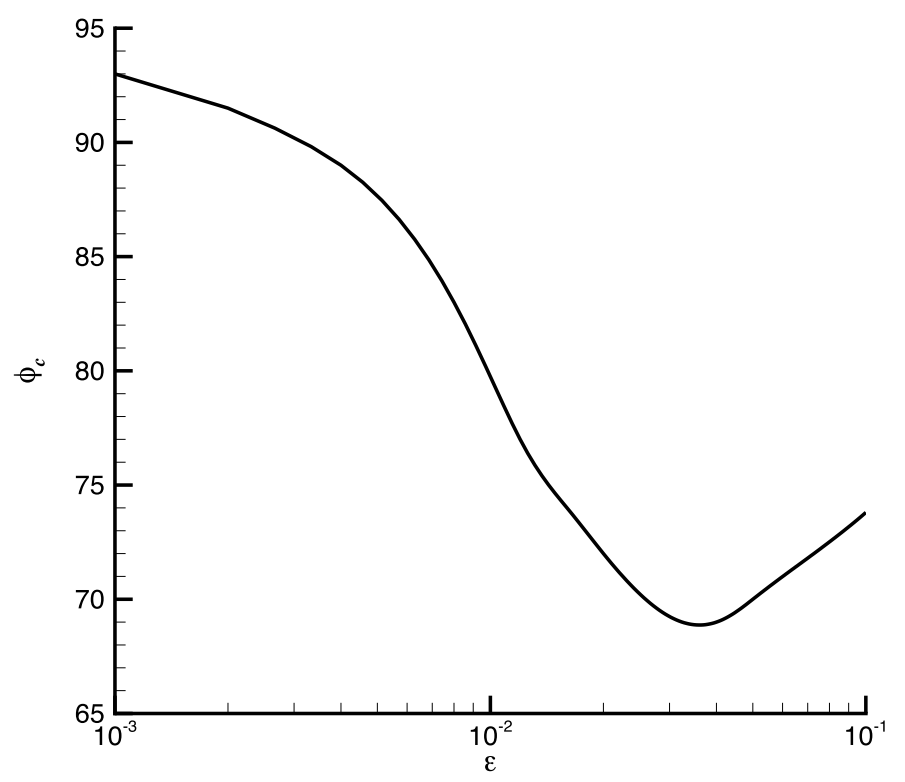

FIG. 8. The wave propagation angle corresponding to the oblique wave in Fig. 6.

\section{The perturbation flow field}

In Table $\mathrm{V}$, we list the growth rate of the spanwise stationary mode for different wave numbers near the critical Marangoni number. It suggests that the growth rates for small wave numbers are very close at the critical condition. Although the critical wave number tends to 0 , the growth rate increases with the wave number when the Marangoni number is a little above the critical value. Additionally, the perturbation flow fields for small wave numbers are all similar. Therefore, we plot the perturbation flow fields at $k=0.1$, for instance, in the following.

In Figs. 18-20, the streamlines and isothermals for spanwise stationary mode and oblique wave are plotted in the direction of wave propagation. The streamlines show that the rolls are arranged periodically for both the modes. For the latter, the rolls have some asymmetric deformity in direction of wave propagation, which is similar to the case for Newtonian fluid. ${ }^{22,36}$ The isothermals



FIG. 9. The wave speed corresponding to the oblique wave and streamwise wave in Fig. 6. 


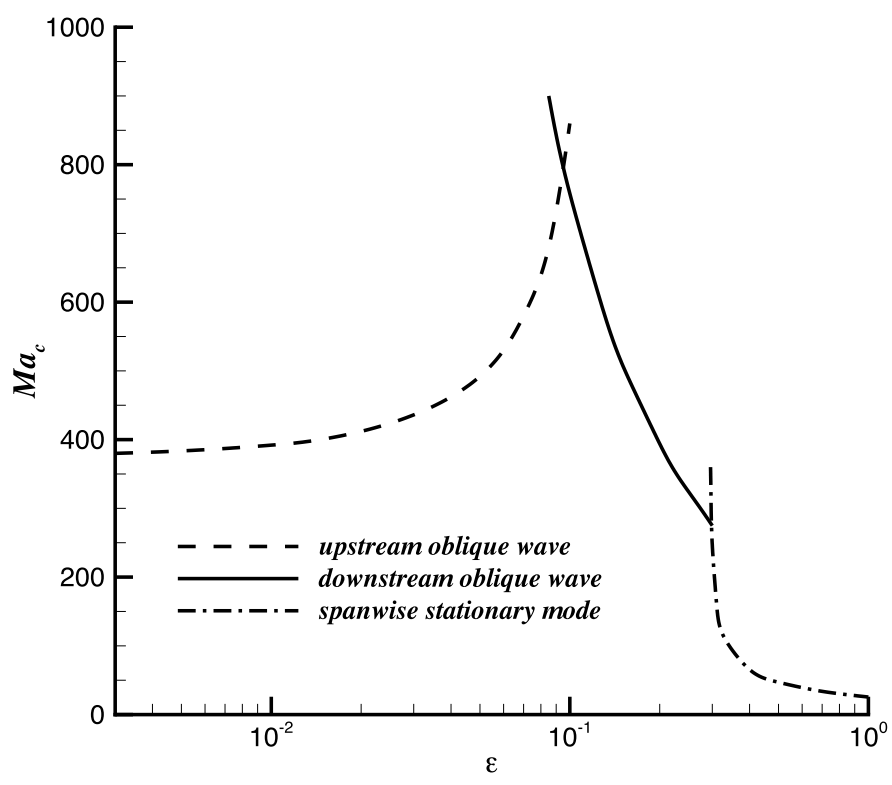

FIG. 10. The variation of $M a_{c}$ with $\varepsilon$ for return flow at $\operatorname{Pr}=100, \eta / \lambda=0.1$.

form many shapes in different parameters and flows. For spanwise stationary mode, they are nearly vertical. For the oblique wave, the location of perturbation amplitude can be on the free surface for $P r=0.02$ (Fig. 19(b)) and in the interior for $P r=100$ (Fig. 20(b)).

However, the case for streamwise wave is distinct from previous two modes. The streamwise velocity $u$ fluctuates several times in the vertical direction in linear flow (see Fig. 21), which do not exist for the streamwise wave in the Newtonian fluid. ${ }^{36} \mathrm{~A}$ similar result is observed for the streamwise wave in return flow.

\section{Energy analysis}

We study the maintenance of perturbation energy in this section. The rate of change for perturbation energy can be written as ${ }^{37,38}$

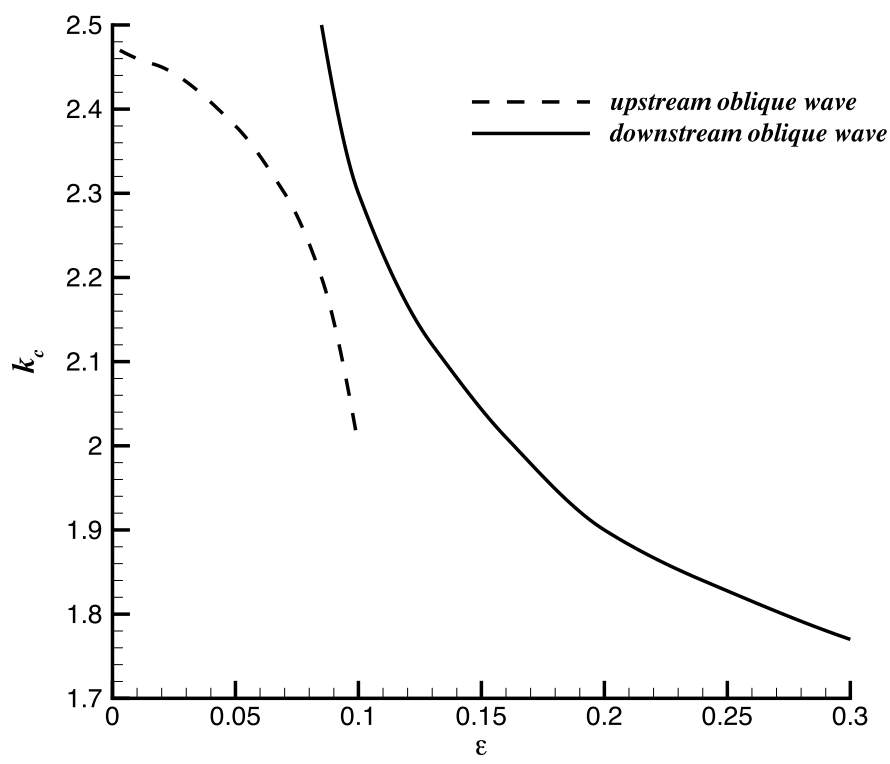

FIG. 11. The wave number corresponding to the upstream and downstream oblique waves in Fig. 10. 


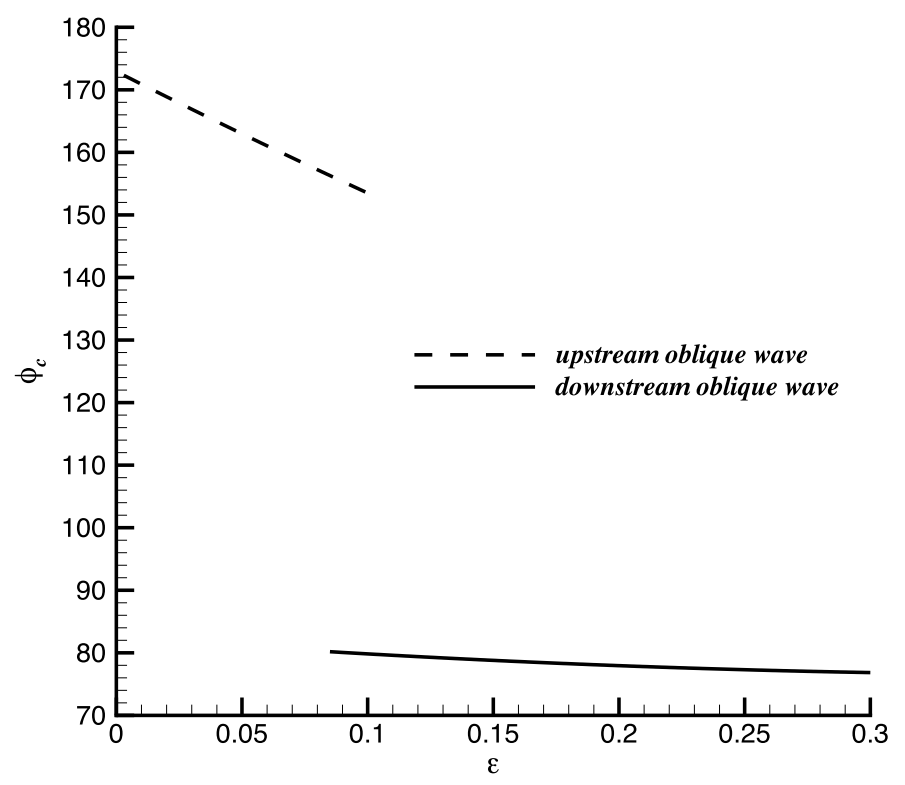

FIG. 12. The wave propagation angle corresponding to the upstream and downstream oblique waves in Fig. 10.

$\frac{\partial E_{k i n}}{\partial t}=-\frac{1}{2 R} \int(\mathbf{Q}: \mathbf{S}) \mathrm{d}^{3} r+\frac{1}{R} \int \mathbf{u} \cdot \mathbf{Q} \cdot \mathbf{n} \mathrm{d}^{2} r-\int \mathbf{u} \cdot\left((\mathbf{u} \cdot \nabla) \mathbf{u}_{0}\right) \mathrm{d}^{3} r=-N+M+I$,

where $N$ is the work done by the perturbation stress, $M$ is the work done by Marangoni forces on the free surface, $I$ is the interaction between the perturbation flow and the basic flow, respectively. The perturbation is normalized as follows:

$$
\int \mathbf{u}^{2} \mathrm{~d}^{3} r=1
$$

The computation shows that for $\operatorname{Pr}=100, I$ is small enough to be neglected. The perturbation energy only depends on the $M$ and $N$, where $M, N>0$. Physically, this means that the source of perturbation energy comes from the Marangoni force caused by perturbation temperature on the



FIG. 13. The wave speed corresponding to the upstream and downstream oblique waves in Fig. 10. 


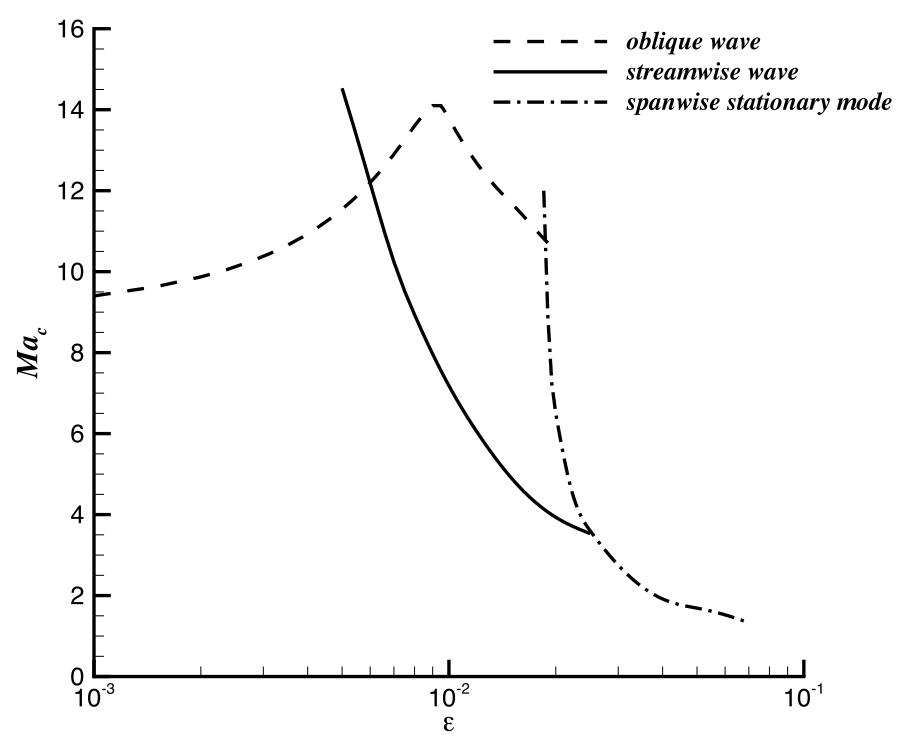

FIG. 14. The variation of $M a_{c}$ with $\varepsilon$ for return flow at $\operatorname{Pr}=0.02, \eta / \lambda=0.1$.

surface, while the perturbation stress is dissipating in the flow region. Compared with Newtonian fluid, it seems that the elasticity does not change the mechanism of perturbation energy significantly.

For $\operatorname{Pr}=0.02$, the energy mechanism changes with mode. In Figs. 22 and 23, we plot the ratios of three terms in (3.1) for the spanwise stationary modes and oblique waves in Figs. 6 and 14.

For spanwise stationary mode, $I$ can also be neglected, which is completely opposite to the case of the stationary mode in liquid bridge for Newtonian fluid at $\operatorname{Pr}=0.02 .{ }^{38}$ For the latter, $I$ is dominant while $M$ is negligible. Physically, the stationary mode for the former is caused by the perturbation temperature on the surface while for the latter, the instability mechanism is purely hydrodynamic.

For oblique wave, the energy coming from the Marangoni force and basic flow balances the dissipation caused by the perturbation stress. Here, $M, I, N>0$. When the oblique wave is the preferred mode, the ratio of $M$ to $I$ is larger than 1 , although it decreases with $\varepsilon$.

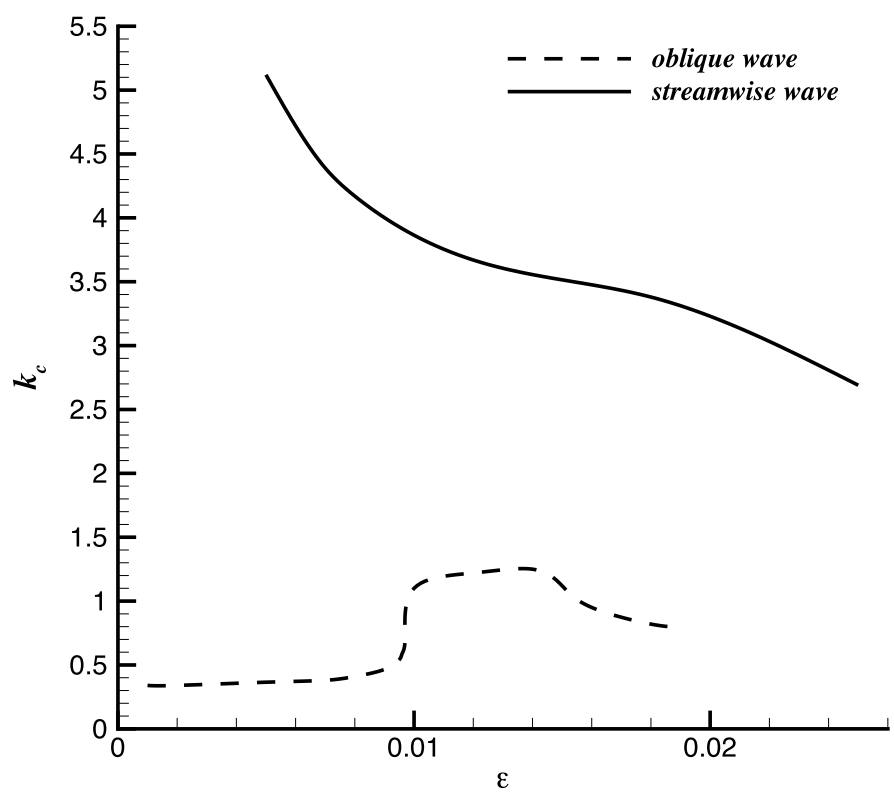

FIG. 15. The wave number corresponding to the oblique wave and streamwise wave in Fig. 14. 




FIG. 16. The wave propagation angle corresponding to the oblique wave in Fig. 14.

For streamwise wave, we list three terms in (3.1) for various cases in Table VI. Each term can change sign in different parameters. The perturbation stress can either dissipate $(N>0)$ or input energy $(N<0)$, which is very distinct from that for the Newtonian fluid. This is due to the phase difference between $\mathbf{Q}$ and $\mathbf{S}$ caused by elasticity. ${ }^{37,39}$

As there are many fluctuations for the perturbation velocity in vertical direction (see Fig. 21), we plot the distribution of the work done by perturbation stress in vertical direction and make comparison between three modes (see Fig. 24). Here,

$$
-N=\int P_{\sigma} \mathrm{d} z, \quad P_{\sigma}=-\frac{1}{2 R} \int(\mathbf{Q}: \mathbf{S}) \mathrm{d}^{2} r .
$$

$P_{\sigma}<0$ stands for the energy dissipation, which always holds for the Newtonian fluid. $P_{\sigma}>0$ stands for the energy input. It can be found that $P_{\sigma}<0$ for oblique wave and spanwise stationary mode. However, for streamwise wave, $P_{\sigma} \approx 0$ when $z<0.5$ while it fluctuates several times in the rest of the region.

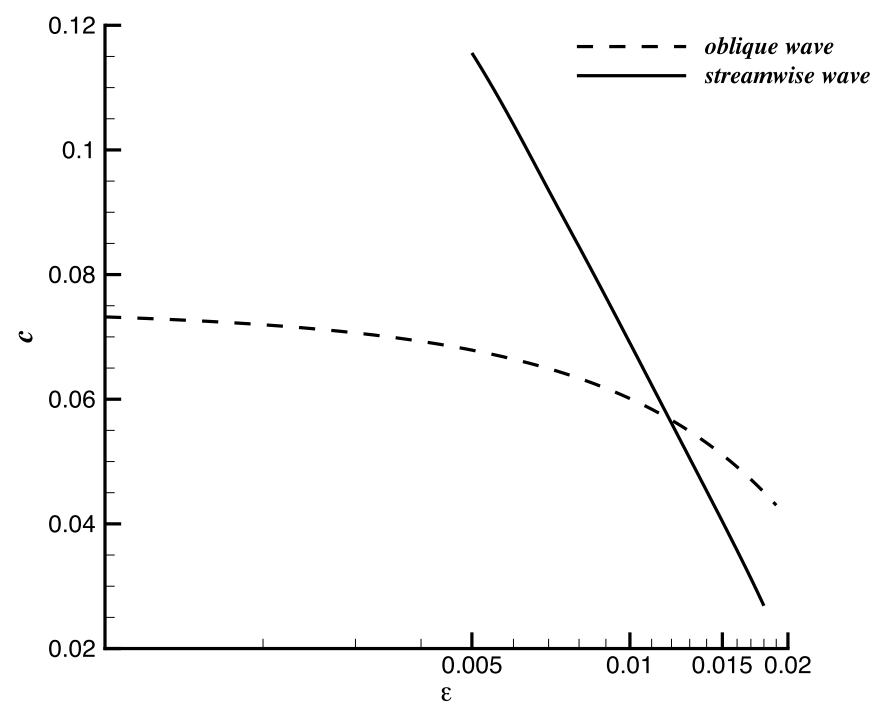

FIG. 17. The wave speed corresponding to the oblique wave and streamwise wave in Fig. 14. 
TABLE V. The growth rate of the spanwise stationary mode for different wave numbers near the critical Marangoni numbers at $\operatorname{Pr}=100, \varepsilon=2$, $\eta / \lambda=0.1$.

\begin{tabular}{lrrrr}
\hline \hline & $k=0.2$ & $k=0.1$ & $k=0.06$ & \multicolumn{1}{c}{$k=0.04$} \\
\hline$M a=11.1$ & 0.000011 & 0.000008 & 0.000003 & 0.000002 \\
$M a=11.0$ & -0.000055 & -0.000008 & -0.000002 & -0.000001 \\
\hline \hline
\end{tabular}

\section{DISCUSSION}

We will discuss the instability mechanism for the Oldroyd-B fluid in this section.

\section{A. Spanwise stationary mode}

The stationary mode for Oldroyd-B fluid only appears in spanwise direction. Its energy comes from the Marangoni force. These features are the same as those in the Newtonian fluid. However, the mechanism has completely changed for high elasticity.

For the Newtonian fluid, the stationary mode in linear flow involves the classical Marangoni instability for the layer being heated from below while the vertical temperature distribution in return flow corresponds to the layer being cooled from below. ${ }^{22}$ Therefore, the stationary instability cannot be found in the latter.

For the Oldroyd-B fluid, when a hot point is on the surface, its heating comes from the heat convection and losses by heat conduction. For $\operatorname{Pr}=0.02$, when the spanwise stationary mode becomes the preferred mode, our computation shows that the terms in (2.10e) have $\left|\widehat{u} \frac{\partial T_{0}}{\partial x}\right| \gg\left|\widehat{w} \frac{\partial T_{0}}{\partial z}\right|$ for $z>0$. The streamwise velocity $u>0$ when underneath the hot spot on the surface, which is just opposite to the case in Newtonian fluid. ${ }^{22}$ It can produce a convective heating and destabilize the flow. These indicate that the horizontal basic-state temperature field transfers heat to the hot point

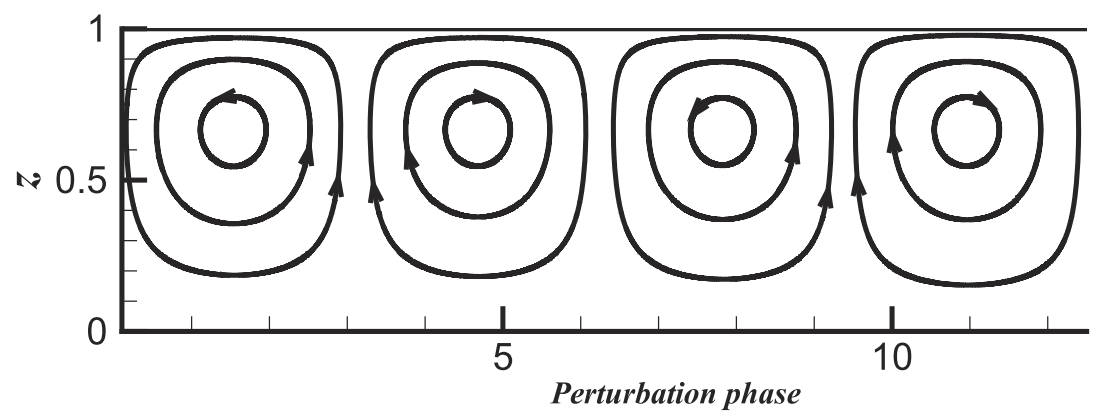

(a)

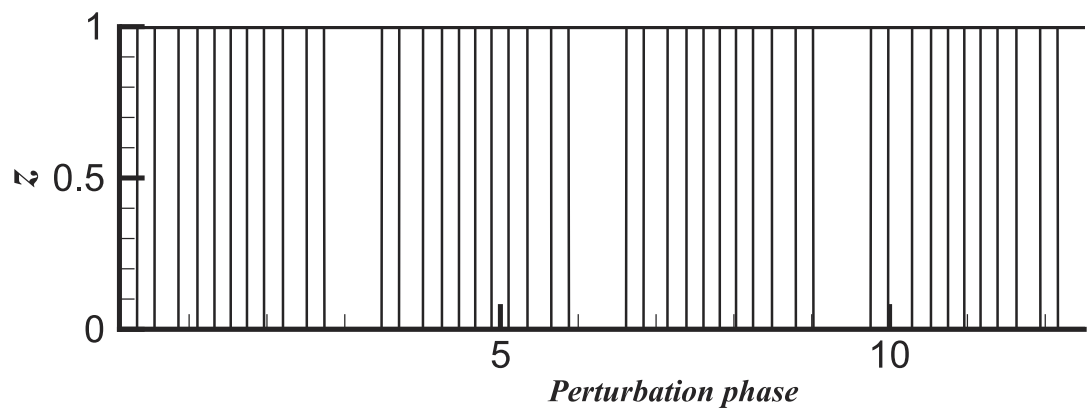

(b)

FIG. 18. The perturbation flow field of the spanwise stationary mode for linear flow at $\operatorname{Pr}=100, \operatorname{Ma}=11.1, k=0.1, \varepsilon=2$, $\eta / \lambda=0.1$ : (a) the streamlines, (b) the isothermals. 


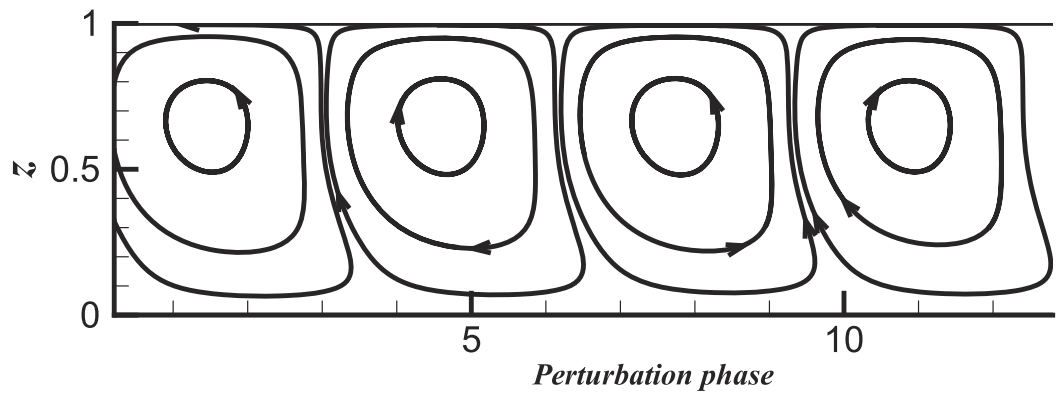

(a)

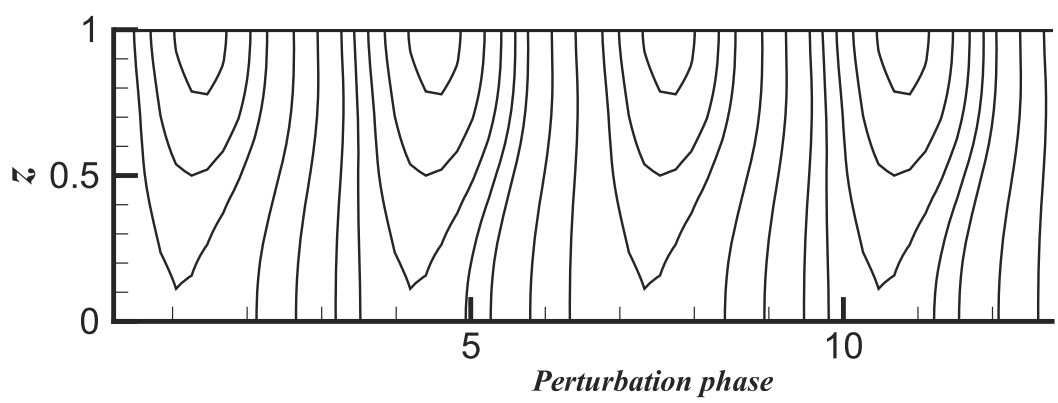

(b)

FIG. 19. The perturbation flow field of the oblique wave for return flow at $\operatorname{Pr}=0.02, M a=11.96, k=1.25, \phi=70^{\circ}$, $\varepsilon=0.014, \eta / \lambda=0.1$ : (a) the streamlines, (b) the isothermals.

through horizontal convection. The vertical temperature distribution is not important for the mechanism. Therefore, the stationary instability appears in both linear flow and return flow, although their vertical temperature gradient is opposite. For $\operatorname{Pr}=100$, the vertical heat convection becomes larger, but still does not affect the mechanism qualitatively.

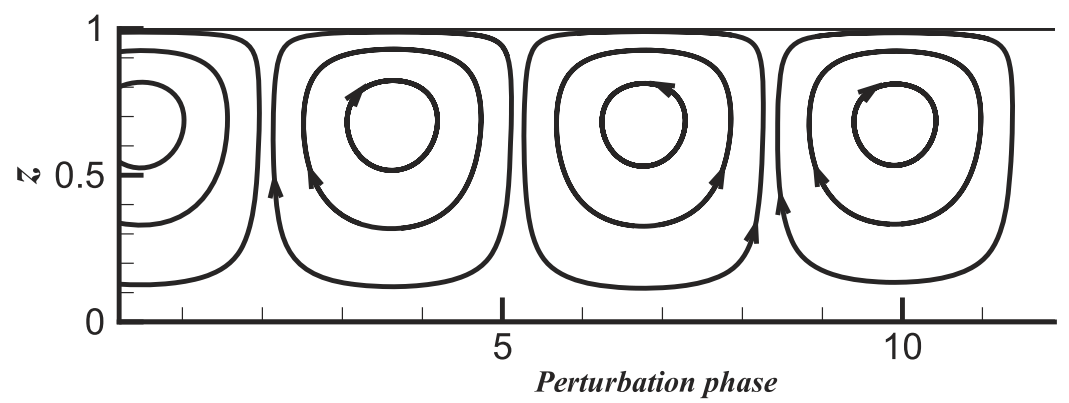

(a)

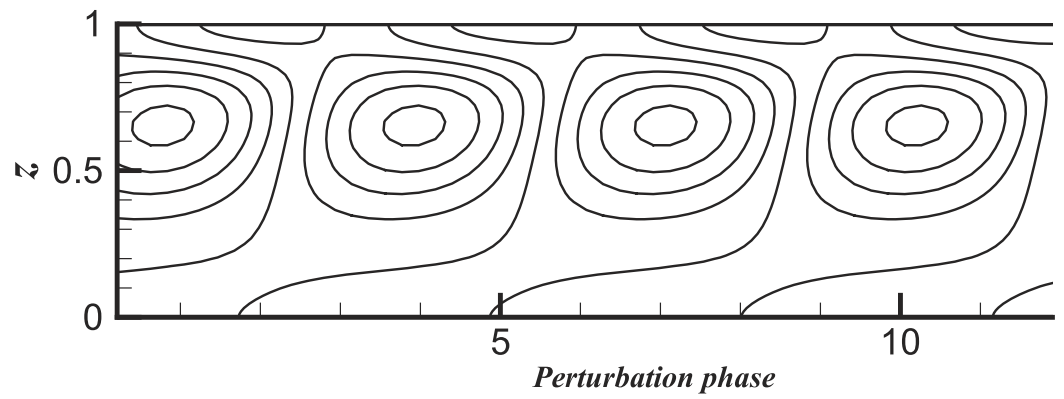

(b)

FIG. 20. The perturbation flow field of the oblique wave for return flow at $\operatorname{Pr}=100, M a=394, k=1.9, \phi=78^{\circ}, \varepsilon=0.2$, $\eta / \lambda=0.1$ : (a) the streamlines, (b) the isothermals. 




FIG. 21. One cycle of the streamwise velocity for streamwise wave. The basic flow is linear flow at $\operatorname{Pr}=0.02, M a=3.49$, $k=2.6, \varepsilon=0.02, \eta / \lambda=0.1$.

The critical wave number of spanwise stationary mode tends to zero. This may be due to the heat conduction. The isothermals are nearly vertical (see Fig. 18(b)), so the heat conduction of perturbation is mainly horizontal. Decreasing wave number also decreases the horizontal temperature gradient, which reduces the heat conduction. For a hot point on the surface, the reduction of

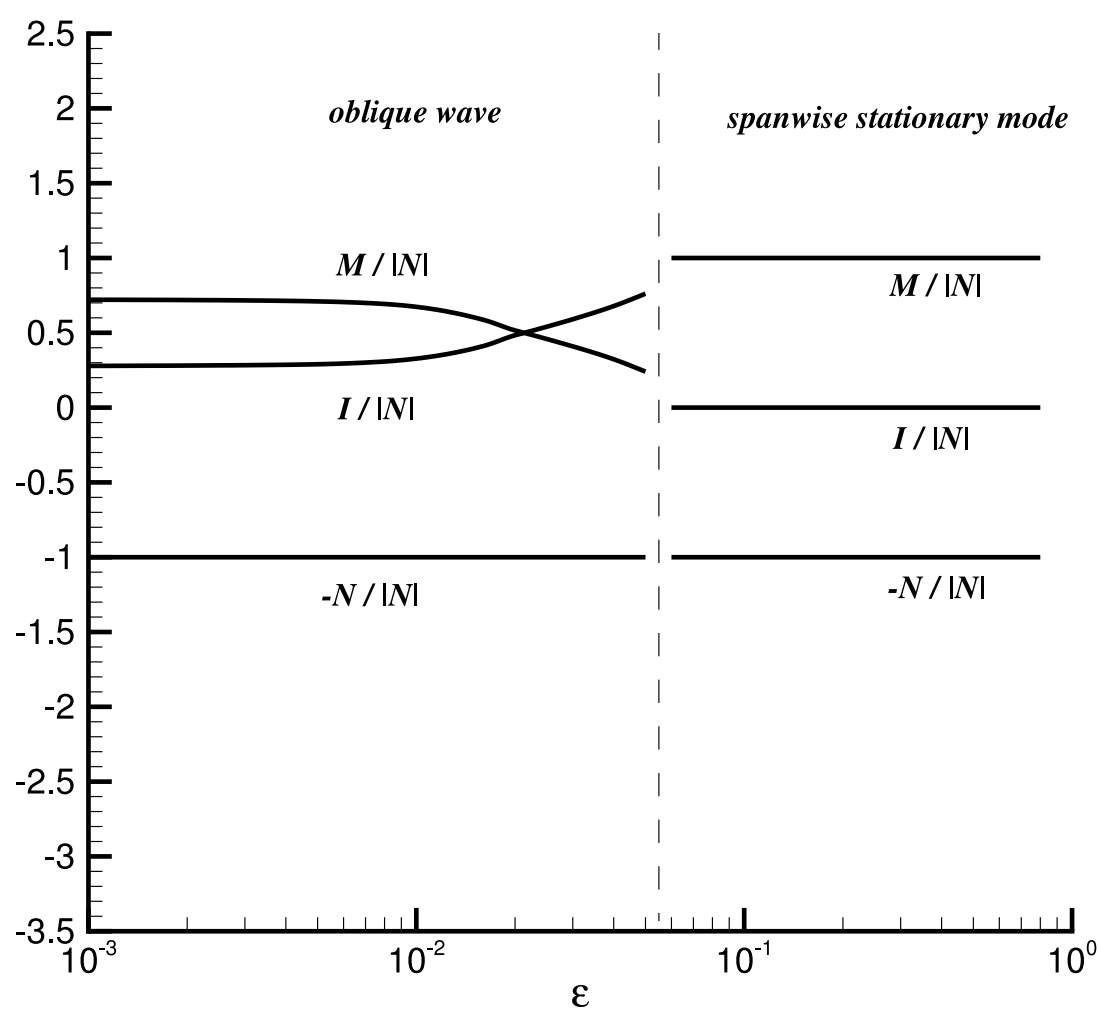

FIG. 22. The ratios of three terms in (3.1) for the oblique wave and spanwise stationary mode of linear low in Fig. 6. 


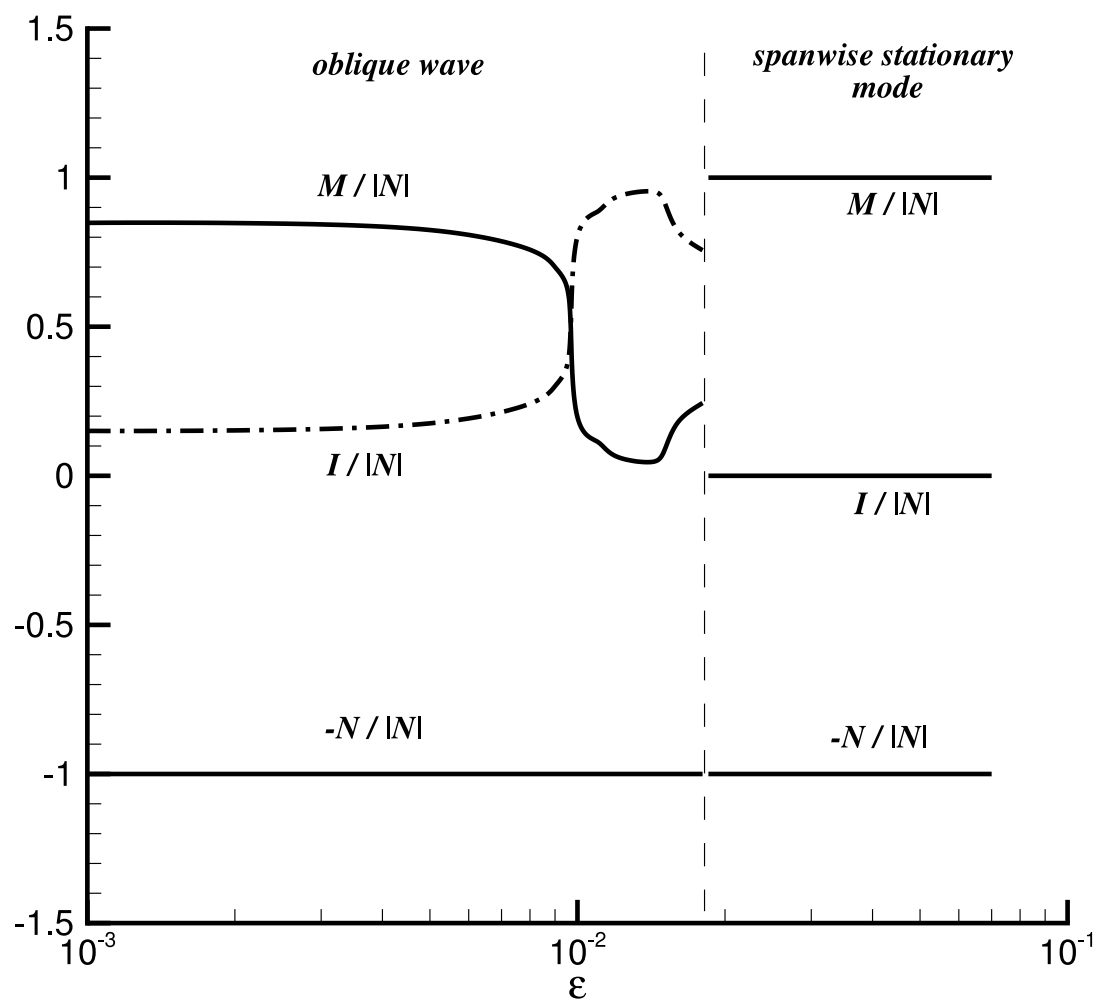

FIG. 23. The ratios of three terms in (3.1) for the oblique wave and spanwise stationary mode of return low in Fig. 14.

conduction can increase its perturbation temperature. Therefore, the flow is more unstable for small wave numbers.

\section{B. Streamwise wave}

For streamwise wave, energy analysis shows that each term in (3.1) can change sign in different flows and parameters. The perturbation stress can input energy to the perturbation because of elasticity. The distributions of $P_{\sigma}$ and $u$ fluctuate in vertical direction. These features are so different from not only the case for Newtonian fluid, but also the other two modes in Oldroyd-B fluid. It seems that the elasticity is more essential than the perturbation temperature on the surface for the case of streamwise wave.

When $\varepsilon$ is moderate, the streamwise wave is the preferred mode for both the linear flow and return flow at $\operatorname{Pr}=0.02$ (see Figs. 6 and 14). In contrast, for $\operatorname{Pr}=100$, it is not preferred at any $\varepsilon$. It is worth noting that the Weissenberg number $\lambda \geq 3$ for the streamwise wave at $\operatorname{Pr}=0.02$ in Figs. 6 and 14, while $\lambda<1$ for the preferred mode at $\operatorname{Pr}=100, \eta / \lambda=0.1$. This suggests that the mechanism of streamwise wave is related to the Weissenberg number, which can reflect the elastic effect in the flow, while the basic flow is of little importance.

TABLE VI. The terms of perturbation energy growth for unstable streamwise wave at $\operatorname{Pr}=0.02, \eta / \lambda=0.1$. Here, LF stands for linear flow while RF stands for return flow.

\begin{tabular}{lrrr}
\hline \hline & \multicolumn{1}{c}{$-N$} & \multicolumn{1}{c}{$M$} & $I$ \\
\hline LF: $M a=5.42, k=3.2, \varepsilon=0.012$ & 0.007468 & 0.013922 & -0.021252 \\
LF: $M a=1.74, k=1.7, \varepsilon=0.05$ & 0.036031 & 0.002713 & -0.038674 \\
RF: $M a=14.53, k=5.12, \varepsilon=0.005$ & 0.003026 & -0.005578 & 0.002555 \\
RF: $M a=8.94, k=4.17, \varepsilon=0.008$ & -0.008220 & -0.004896 & 0.013125 \\
RF: $M a=4.22, k=3.38, \varepsilon=0.018$ & 0.006761 & -0.002381 & -0.004376 \\
\hline \hline
\end{tabular}




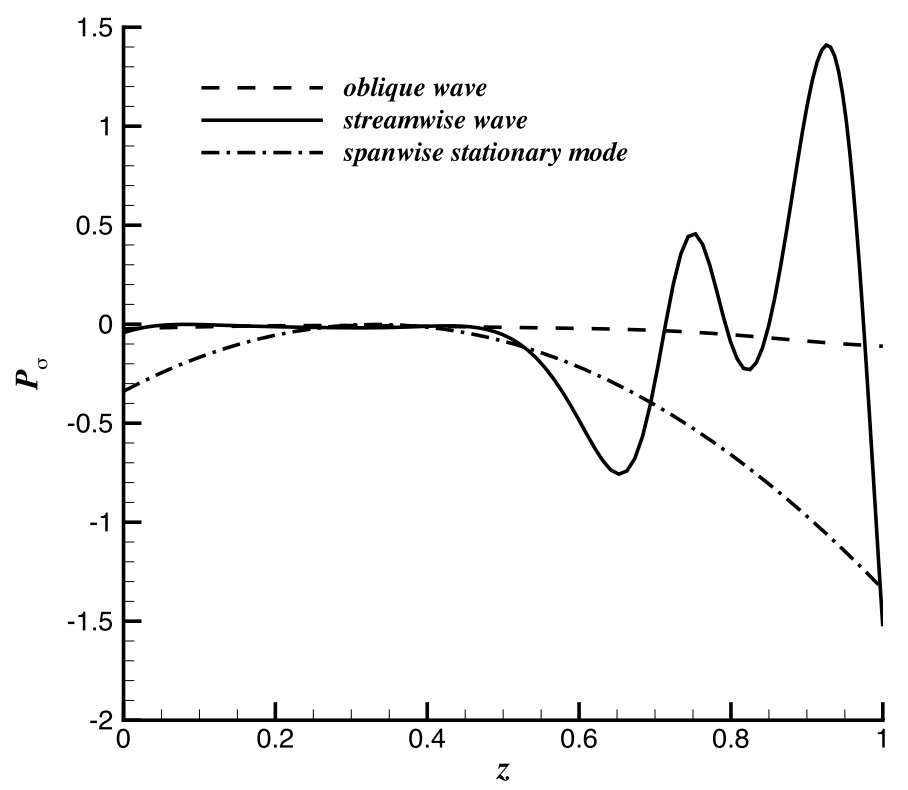

FIG. 24. The distribution of the work done by perturbation stress in vertical direction at $\operatorname{Pr}=0.02, \eta / \lambda=0.1$. The parameters for each mode are (a) oblique wave: $M a=11.54, k=0.365, \theta=85^{\circ}, \varepsilon=0.005$; (b) streamwise wave: $M a=4.22, k=3.38$, $\varepsilon=0.018 ;$ (c) spanwise stationary mode: $M a=1.69, k=0.03, \varepsilon=0.05$.

\section{Oblique wave}

For the oblique wave, $M a_{c}$ increases with the $\varepsilon$ when $\varepsilon$ is small. The explanation is as follows. In this region, the work done by Marangoni force is the main source for perturbation energy. For the Newtonian fluid, the Marangoni force keeps the same phase with the perturbation velocity on the free surface. However, there is a phase difference between them for Oldroyd-B fluid because of elasticity. Thus, the work efficiency of Marangoni force decreases, and the flow becomes more stable. A higher Marangoni number is needed to increase the energy input by Marangoni force and move the flow back to the neutral point.

The increasing of Marangoni number increases the inertia of basic flow, which also affects the oblique wave. Then its wave speed in streamwise direction increases. Therefore, the propagation angle of oblique wave decreases with $\varepsilon$ when $\varepsilon$ is small.

\section{CONCLUSION}

The linear stability of thermocapillary liquid layers for Oldroyd-B fluid has been investigated. Results have been obtained for linear flow and return flow with Prandtl numbers of 0.02 and 100, which shows that the elasticity has a great impact on the critical parameters, critical mode, and perturbation mechanism.

Elastic mode still exists for Oldroyd-B fluid. However, it decays rapidly with $\eta / \lambda$ and does not affect the stability for $\eta / \lambda \geq 0.1$. The increasing of $\eta / \lambda$ always makes the flow stability approach to that for Newtonian fluid.

For linear flow, when $\operatorname{Pr}=100$, the preferred is the spanwise stationary mode. Its critical wave number tends to zero and $M a_{c}$ decreases with $\varepsilon$. When $\operatorname{Pr}=0.02$, with the increasing of $\varepsilon$, the preferred modes are oblique wave, streamwise wave, and spanwise stationary mode in that order. For the first mode, $M a_{c}$ increases slightly with $\varepsilon$ and the wave changes from upstream to downstream. For the last two modes, $M a_{c}$ decreases with $\varepsilon$ significantly.

For return flow, when $\operatorname{Pr}=100$, with the increasing of $\varepsilon$, the preferred modes are upstream oblique wave, downstream oblique wave, and spanwise stationary mode in that order. For the first mode, $M a_{c}$ increases with $\varepsilon$ continuously while for the last two modes, $M a_{c}$ decreases with $\varepsilon$ swiftly. When $\operatorname{Pr}=0.02$, the variation of the preferred mode with $\varepsilon$ is similar to the case of linear flow. 
The streamlines for spanwise stationary mode and oblique wave are both periodically arranged rolls while the perturbation velocity fluctuates several times in vertical direction for streamwise wave. The isothermals have several shapes in different cases. For spanwise stationary mode, they are nearly vertical, while for oblique wave, the location of perturbation amplitude can be either on the free surface or in the interior of flow region.

Energy analysis shows that when $P r=100$, the perturbation energy comes from the Marangoni force caused by perturbation temperature while dissipates by perturbation stress. The impact of basic flow is negligible. When $\operatorname{Pr}=0.02$, the energy mechanism is different for three modes. The case for spanwise stationary mode is similar to that at $\operatorname{Pr}=100$. For oblique wave, the perturbation energy comes from the Marangoni force and the basic flow. For streamwise wave, the work done by perturbation stress fluctuates in the vertical direction.

Comparing with the Newtonian fluid, it is found that the weak elasticity makes the oblique wave more stable while the strong elasticity is always destabilizing. The preferred mode and its propagation direction are changed significantly by elasticity. A distinct difference is that the stationary instability does not exist in return flow for Newtonian fluid, however, when $\varepsilon$ is high enough, the spanwise stationary mode becomes the preferred mode for Oldroyd-B fluid in both linear flow and return flow. Although the three modes for Oldroyd-B fluid also appear in the Newtonian fluid, their mechanisms are greatly affected by elasticity.

\section{ACKNOWLEDGMENTS}

This work has been supported by the National Natural Science Foundation of China (Nos. 11272320, 11402271, and 11532015).

${ }^{1}$ Crystal Growth Processes Based on Capillarity: Czochralski, Floating Zone, Shaping and Crucible Techniques, edited by T. Duffar (John Wiley \& Sons, 2010).

${ }^{2}$ M. F. Schatz and G. P. Neitzel, "Experiments on thermocapillary instabilities,” Annu. Rev. Fluid Mech. 33(1), 93-127 (2001).

${ }^{3}$ R. J. Riley and G. P. Neitzel, "Instability of thermocapillary-buoyancy convection in shallow layers. Part 1. Characterization of steady and oscillatory instabilities," J. Fluid Mech. 359, 143-164 (1998).

${ }^{4}$ T. DebRoy and S. A. David, "Physical processes in fusion welding," Rev. Mod. Phys. 67(1), 85-112 (1995).

${ }^{5}$ K. C. Mills, B. J. Keene, R. F. Brooks, and A. Shirali, “Marangoni effects in welding,” Philos. Trans. R. Soc., A 356, 911-925 (1998).

${ }^{6}$ S. W. Pierce, P. Burgardt, and D. L. Olson, “Thermocapillary and arc phenomena in stainless steel welding,” Weld. J. 78, 45-52 (1999), see www.aws.org/wj/supplement/Pierce/ARTICLE3.pdf.

${ }^{7}$ W. A. Sirignano and I. Glassman, "Flame spreading above liquid fuels: Surface-tension-driven flows," Combust. Sci. Technol. 1(4), 307-312 (1970).

${ }^{8}$ S. H. Davis, “Thermocapillary instabilities," Annu. Rev. Fluid Mech. 19(1), 403-435 (1987).

${ }^{9}$ M. J. Troughton, Handbook of Plastics Joining: A Practical Guide (William Andrew, 2008).

${ }^{10} \mathrm{~J}$. Rotheiser, Joining of Plastics (Hanser, 1999).

${ }^{11}$ D. Grewell and A. Benatar, "Welding of plastics: Fundamentals and new developments," Int. Polym. Process. 22(1), 43-60 (2007).

12 J. J. Chen and J. D. Lin, “Thermocapillary effect on drying of a polymer solution under non-uniform radiant heating," Int. J. Heat Mass Transfer 43(12), 2155-2175 (2000).

${ }^{13}$ G. Toussaint, H. Bodiguel, F. Doumenc, B. Guerrier, and C. Allain, "Experimental characterization of buoyancy-and surface tension-driven convection during the drying of a polymer solution," Int. J. Heat Mass Transfer 51(17), 4228-4237 (2008).

${ }^{14}$ M. Yamamura, S. Wajima, Y. Mawatari, and H. Kage, "Nonuniform thinning of polymeric coatings under Marangoni stress," J. Chem. Eng. Jpn. 43(1), 40-45 (2010).

${ }^{15}$ G. T. Dee and B. B. Sauer, "The surface tension of polymer liquids," Adv. Phys. 47(2), 161-205 (1998).

${ }^{16}$ D. L. Hitt and M. K. Smith, "Radiation-driven thermocapillary flows in optically thick liquid films," Phys. Fluids A 5(11), 2624-2632 (1993).

${ }^{17}$ E. B. Guto, E. D. Cohen, and G. I. Kheboian, Coating and Drying Defects (Wiley, New York, 1995).

${ }^{18}$ R. B. Bird, C. F. Curtiss, R. C. Armstrong, and O. Hassager, Dynamics of Polymeric Liquids: Kinetic Theory (John Wiley \& Sons, 1987), Vol. 2.

${ }^{19}$ R. R. Huilgol and N. Phan-Thien, Fluid Mechanics of Viscoelasticity: General Principles, Constitutive Modelling, Analytical and Numerical Techniques (Elsevier, 1997).

${ }^{20}$ R. G. Larson, The Structure and Rheology of Complex Fluids (Oxford University Press, 1999).

${ }^{21}$ N. Hoda, M. R. Jovanovic, and S. Kumar, “Energy amplification in channel flows of viscoelastic fluids,” J. Fluid Mech. 601, 407-424 (2008).

${ }^{22}$ M. K. Smith and S. H. Davis, "Instabilities of dynamic thermocapillary liquid layers. Part 1. Convective instabilities," J. Fluid Mech. 132, 119-144 (1983).

${ }^{23}$ D. Getachew and S. Rosenblat, "Thermocapillary instability of a viscoelastic liquid layer," Acta Mech. 55(1-2), 137-149 (1985). 
${ }^{24}$ P. C. Dauby, P. Parmentier, G. Lebon, and M. Grmela, "Coupled buoyancy and thermocapillary convection in a viscoelastic Maxwell fluid," J. Phys.: Condens. Matter 5(26), 4343 (1993).

${ }^{25}$ G. Lebon, P. Parmentier, O. Teller, and P. C. Dauby, "Bénard-Marangoni instability in a viscoelastic Jeffreys' fluid layer," Rheol. Acta 33(4), 257-266 (1994).

${ }^{26}$ P. Parmentier, G. Lebona, and V. Regnier, "Weakly nonlinear analysis of Benard-Marangoni instability in viscoelastic fluids," J. Non-Newtonian Fluid Mech. 89(1), 63-95 (2000).

${ }^{27}$ H. Ramkissoon, G. Ramdath, D. Comissiong, and K. Rahaman, "On thermal instabilities in a viscoelastic fluid," Int. J. Non-Linear Mech. 41(1), 18-25 (2006).

${ }^{28}$ P. G. Siddheshwar, G. N. Sekhar, and G. Jayalatha, "Surface tension driven convection in viscoelastic liquids with thermorheological effect," Int. Commun. Heat Mass Transfer 38(4), 468-473 (2001).

${ }^{29}$ I. Pérez-Reyes and L. A. Dávalos-Orozco, "Effect of thermal conductivity and thickness of the walls in the convection of a viscoelastic Maxwell fluid layer,” Int. J. Heat Mass Transfer 54(23), 5020-5029 (2011).

${ }^{30}$ P. N. Kaloni and J. X. Lou, "On the convective stability of Oldroyd B fluid subject to a horizontal temperature gradient," in ASME/JSME 2003 4th Joint Fluids Summer Engineering Conference, Honolulu, Hawaii, USA, 6-10 July 2003 (American Society of Mechanical Engineers, 2003), pp. 1601-1606.

${ }^{31}$ K. X. Hu, M. He, and Q. S. Chen, "The linear stability of thermocapillary liquid layers for UCM fluid," J. Non-Newton. Fluid Mech. (submitted).

${ }^{32}$ K. R. Rajagopal and R. K. Bhatnagar, "Exact solutions for some simple flows of an Oldroyd-B fluid,” Acta Mech. 113(1-4), 233-239 (1995).

${ }^{33}$ R. Sureshkumar and A. Beris, "Linear stability analysis of viscoelastic Poiseuille flow using an Arnoldi-based orthogonalization algorithm," J. Non-Newtonian Fluid Mech. 56, 151-182 (1995).

${ }^{34}$ Y. R. Li, N. Imaishi, T. Azami, and T. Hibiya, "Three-dimensional oscillatory flow in a thin annular pool of silicon melt," J. Cryst. Growth 260(1), 28-42 (2004).

${ }^{35}$ Y. R. Li, N. Imaishi, L. Peng, S. Y. Wu, and T. Hibiya, "Thermocapillary flow in a shallow molten silicon pool with Czochralski configuration,” J. Cryst. Growth 266(1), 88-95 (2004).

${ }^{36}$ M. K. Smith, "Instability mechanisms in dynamic thermocapillary liquid layers," Phys. Fluids 29(10), 3182-3186 (1986).

${ }^{37}$ K. X. Hu, J. Peng, and K. Q. Zhu, "The linear stability of plane Poiseuille flow of Burgers fluid at very low Reynolds numbers," J. Non-Newtonian Fluid Mech. 167-168, 87-94 (2012).

${ }^{38}$ M. Wanschura, V. M. Shevtsova, H. C. Kuhlmann, and H. J. Rath, "Convective instability mechanisms in thermocapillary liquid bridges," Phys. Fluids 7(5), 912-925 (1995).

${ }^{39}$ K. X. Hu, J. Peng, and K. Q. Zhu, "Linear stability of plane creeping Couette flow for Burgers fluid," Acta Mech. Sin. 29, 12-23 (2013). 